\title{
Panel M.T.C. * Experimentación sobre un sistema constructivo para viviendas rurales en Boyacá, Colombia
}

\author{
Panel M.T.C. Building system for rural houses in the \\ region of Boyacá, Colombia \\ Panel M.T.C. Sistema costruttivo per case rurali nella \\ regione di Boyacá, Colombia
}

\author{
Laura Beaudu ${ }^{* * *}$ y Fiammetta Conforti****
}

Citar este artículo como: Beaudu, Laura y Conforti, Fiammetta. (2017). Panel M.T.C. - Experimentación sobre un sistema constructivo para viviendas rurales en Boyacá, Colombia. Revista Nodo, 12(23), 38-48

\section{Resumen}

Este artículo muestra el proceso de investigación y realización del Panel M.T.C. como técnica constructiva para viviendas en zonas rurales de Boyacá (Colombia). La edificación de viviendas en este contexto es algo delicado ya que se trata de zonas alejadas y caracterizadas por una gran pobreza. Actualmente se ha perdido e 1 uso de las técnicas constructivas tradicionales, adoptando soluciones "modernas" inadecuadas al contexto y costosas (materiales convencionales). La propuesta del Panel M.T.C. se fundamenta en la reinterpretación del bahareque ${ }^{1}$ y permite usar recursos locales (madera, tierra cruda, caña brava) y conocimientos ancestrales relacionados al contexto rural boyacense. El proyecto se articuló en dos fases, una de estudio del contexto socio-cultural y otra de investigación sobre las técnicas constructivas. Por ello se organizó en Ráquira (Boyacá) un taller con artesanos y algunas entrevistas a los habitantes. De tal manera se desarrolló un panel, cuya modularidad permite la parcial prefabricación y su conclusión a través de procesos de autoconstrucción. La solución permite desarrollar viviendas sostenibles de un piso, respondiendo a las necesidades ambientales, a la población y en acuerdo con el contexto geográfico y cultural. Sin embargo este proyecto abre un camino para profundizar en contextos y situaciones análogas.

Palabras clave: Arquitectura rural colombiana, Construcciones en tierra y caña brava, Sostenibilidad, Identidad cultural, Participación.

Fecha de recepción: 20-05-2016 • Fecha de aceptación: 25-01-2017

\footnotetext{
* M.T.C. La sigla se refiere a los materiales madera, tierra y caña brava (Arundo donax: planta de la familia de las gramíneas).

*: Arquitecta (Grado y máster en arquitectura, Università degli Studi di Firenze. Correo electrónico: 1.beaudu@hotmail.com

*:* Arquitecta (Grado y máster en arquitectura, Università degli Studi di Firenze. Correo electrónico: conforti@gmail.com

1 Bahareque: Sistema de construcción típico de la tradición arquitectónica rural colombiana. Está hecho por una estructura en madera o caña y relleno de barro (Minke, 2010).
} 


\section{Abstract $^{1}$}

This paper describes the process and outcomes of "Panel M.T.C" research as an innovative building system for rural houses in Boyacá (Colombia). Housing in this context is an important issue as it involves remote areas, characterized by extreme poverty. Nowadays, traditional building techniques have been supplanted by more modern solutions although they are hardly applicable in rural areas and unaffordable because they involve imported materials. The proposal of Panel M.T.C. is based on the reinterpretation of bahareque ${ }^{2}$, using local resources (wood, earth, caña brava) and recovering ancestral knowledge related to the local tradition. The research is divided into two parts, one on the socio-cultural context and the other on building techniques. For this purpose, a workshop with artisans was launched in Ráquira (municipality of Boyacá) and some local inhabitants have been interviewed. The panel is modular in order to facilitate prefabrication while the completion occurs by self-construction. This solution achieves flexible one storey housing, where sustainability is key to respond environmental, social and economic needs. Eventually, this project could be applied in comparable contexts of the world with few changes to fit local conditions.

Keywords: Colombian rural architecture, Buildings in cob and caña brava, Sustainability, Tacit knowledge, Cultural identity, Participatory design

\section{Introducción}

El Panel M.T.C. se refiere al producto de una investigación dirigida desde la Universidad de Florencia (Italia) por el arquitecto Antonio Laurìa (profesor de la "Unità di Ricerca Interdipartimentale, Florence Accessibility Lab - Dipartimento di Architettura"). Se trata de un panel portante en madera, tierra cruda y caña brava, basado en la

2 Bahareque: Traditional Colombian building technique. It consists of a structural wooden frame that is filled with raw earth. reinterpretación de la tradición constructiva local. En este artículo se describe el proceso a través del cual se desarrolló este proyecto, desde sus fases iniciales de investigación hasta la definición del producto.

La investigación nació a partir de la constatación de que en Colombia existe la necesidad, además de la posibilidad, de desarrollar intervenciones en construcción y hábitat sostenible. Se llevó a cabo en algunas zonas rurales de Boyacá, rica en tradiciones culturales y en recursos materiales, en donde ha sido posible pensar en el uso de alternativas constructivas con menor impacto ambiental en comparación con aquellas difundidas en las últimas décadas. El propósito fue la realización de un sistema constructivo que permita responder a criterios de sostenibilidad ambiental, económica, social y cultural. El tema de la sostenibilidad ambiental tiene como objetivo el uso adecuado y respetuoso con la ética medioambiental de los recursos. La sostenibilidad económica busca limitar los costos relativos a la edificación y manutención de la obra. La sostenibilidad social trata de relacionarse con la comunidad mediante la participación e implicación de un mayor número de personas. Y finalmente la sostenibilidad cultural busca preservar la identidad local relacionada con la tradición boyacense.

\section{Metodología}

La investigación se desarrolló en dos fases: análisis teórica y experimentación in situ. En la fase teórica se consultó bibliografía científica y se habló con docentes consiguiendo colectar informaciones relativas a este tipo de investigación. Esta fase fue esencial para obtener sólidos conocimientos sobre el contexto cultural y sobre los materiales locales. La fase de experimentación in situ inició con el análisis de aspectos relativos al contexto (geografía, población, economía y cultura) y ha sido caracterizada por la aproximación a las comunidades rurales a través de entrevistas e inspecciones que han brindado el material necesario para entender 
el contexto e interpretar las necesidades de los habitantes.

En este artículo se describe con particular énfasis los aspectos relacionados con el trabajo adelantado con la comunidad de Boyacá que jugó un rol central en el desarrollo de la investigación y en los resultados alcanzados. Además se experimentaron, bajo la guía de maestros, algunas técnicas constructivas basadas en el empleo de tierra cruda y caña brava, hasta obtener un bagaje técnico imprescindible para desarrollar el proyecto del panel.

Hábitat rural e interacción con la comunidad. En el departamento de Boyacá el 44,72\% de la población vive en zonas rurales (Departamento Administrativo Nacional de Estadística,DANE, 2005). Este dato, por su fuerte incidencia, fue el motivo del desarrollo de un proyecto dirigido a la comunidad rural. La estadía en el departamento de Boyacá ha permitido acercarse a un contexto bien distinto al de Italia por medio del estudio de aspectos sociales y culturales encontrados en las costumbres y en la arquitectura.

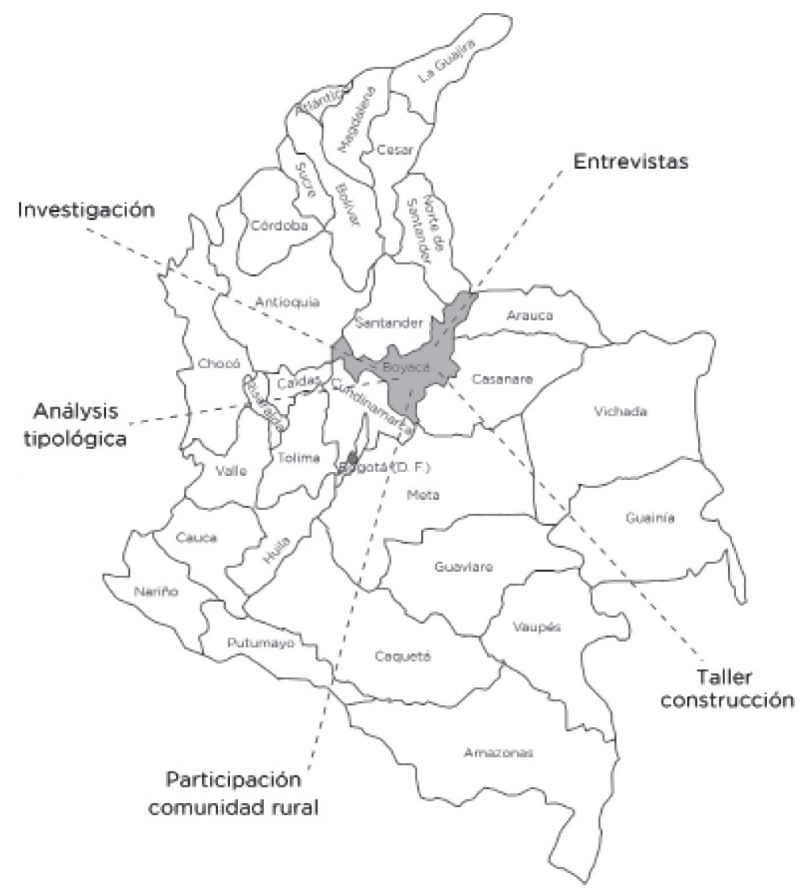

Figura 1. Desarrollo del proyecto en Boyacá. Fuente: propia

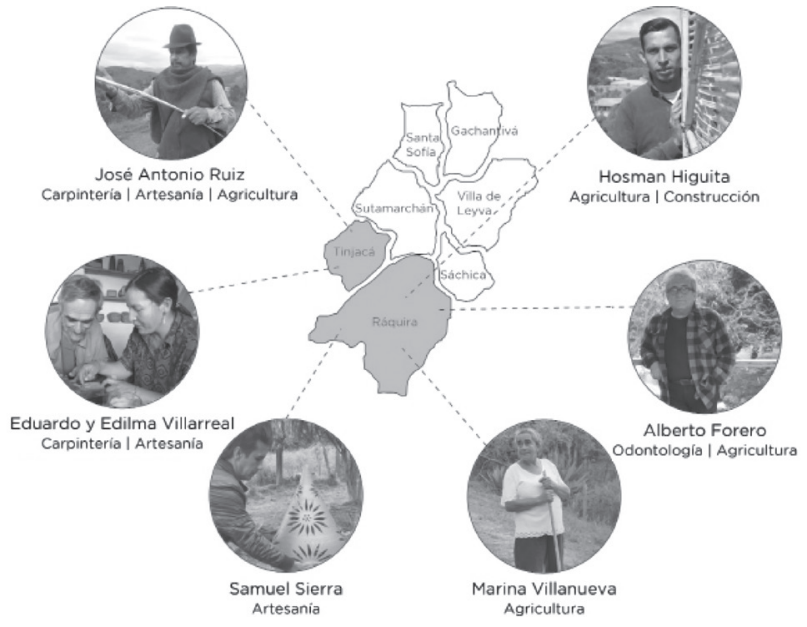

Figura 2. Personas entrevistadas. Fuente: propia

A través de entrevistas, dirigidas y aplicadas a habitantes de la comunidad, se identificaron los requisitos de una casa rural y se evaluaron las soluciones constructivas que alcanzaran un resultado coherente con estos requisitos. Para este fin se elaboró un cuestionario (idéntico en todas las entrevistas) que permitió abordar las siguientes temáticas: uso de los materiales en las casas tradicionales, evaluación de la reintroducción de procesos constructivos tradicionales, comprensión del posible impacto de la introducción del Panel M.T.C., promoción de talleres participativos con los residentes y desarrollo regional mediante el uso de recursos locales.

La selección de las personas entrevistadas se hizo según diferentes criterios. Primero los habitantes que representan a la comunidad, por tener fuerte influencia, empatía y capacidad de involucrar al resto de la gente en la organización y toma de decisiones. Posteriormente aquellos habitantes que, trabajando en el sector de la construcción y siendo especialistas en materiales y técnicas tradicionales, fueron capaces de transmitir sus conocimientos en relación con las diferentes técnicas. Además de obtener conocimientos de carácter técnico, las entrevistas permitieron recabar informaciones complementarias sobre la vida laboral y familiar que definieron las expectativas de los demás habitantes. 
Durante las inspecciones en zonas rurales se encontró que, en la actualidad, no existe un método de construcción único y ampliamente aplicado a lo largo del territorio. De hecho, las casas observadas son en su mayoría hechas por autoconstrucción o por mano de obra no calificada. En otros casos, las intervenciones fueron realizadas por constructores extraños a la región, aplicando métodos y técnicas no relacionadas con el contexto.

Teniendo en cuenta lo anterior el estudio se concentró en las técnicas constructivas tradicionales, de las cuales algunas aún se utilizan, mientras otras han caído en desuso como consecuencia del uso y de la introducción de técnicas innovadoras o provenientes de otras regiones. El estudio ha brindado los elementos para entender los pros y contras de las diversas técnicas y ha permitido determinar los aspectos sobre los cuales intervenir desde la óptica de una posible reintroducción de técnicas tradicionales en la construcción y en el hábitat contemporáneo.

La investigación comprendió también un análisis tipológico de viviendas rurales, evidenciando los espacios típicos y las relativas funciones. Tradicionalmente, las casas tienen forma rectangular o en "L" con un porche frente a uno o más lados. En las construcciones más recientes se ha tendido a ir en la búsqueda de una espacialidad más articulada, con la adición de espacios con diversas o nuevas funciones, retomando a menudo modelos tipológicos urbanos que se difunden con mayor facilidad, debido a la llegada cada vez mayor de personas y medios provenientes de diversas regiones del país (Fonseca Martínez y Saldarriaga Roa, 1992).

Con el fin de realizar el estudio tipológico de las viviendas rurales se han puesto en evidencia algunos aspectos de carácter tecnológico, adoptando la siguiente clasificación:

- Construcciones autóctonas: aquellas realizadas empleando materiales disponibles localmente o en las inmediaciones, y que no requieren procesamiento específico a los efectos de su uso en la construcción. Entre estos materiales está la tierra cruda (trabajada para hacer bahareque o tapia pisada $\left.{ }^{3}\right)$, guadua, caña brava, paja y madera rolliza.

- Construcciones tradicionales: son aquellas realizadas mediante el empleo de materiales derivados del trabajo artesanal local que son característicos de una tradición constructiva en aquel contexto. En Boyacá los materiales de la tradición constructiva son el adobe ${ }^{4}$, los adoquines y diversas maderas.

- Construcciones transitorias: son aquellas que inicialmente fueron autóctonas o tradicionales de las cuales algunas partes han sido sustituidas utilizando materiales modernos. Se puede también tratar de construcciones proyectadas inicialmente integrando técnicas autóctonas, tradicionales y modernas. Se encuentran diversos ejemplos donde se han empleado técnicas tradicionales como el bahareque o el adobe para la mampostería mientras las cubiertas se realizan con materiales modernos como tejas de zinc $u$ onduladas en fibrocemento.

- Construcciones modernas: aquellas realizadas utilizando materiales producidos industrialmente y cuyo aspecto frecuentemente retoma tipologías urbanas.

A partir de esta clasificación se distinguió ulteriormente las categorías de construcciones"típicas" o "atípicas". La primera categoría se refiere a aquellas casas que se identifican con una tipología predominante en la región (en lo que respecta a la morfología, la disposición y la selección de materiales). La segunda se refiere a aquellas casas que presentan ambigüedades en relación a la tipología y la técnica constructiva, en relación con el contexto (Fonseca Martínez, Saldarriaga Roa, 1980), tal como se presenta en la figura 3.

3 Tapia pisada: procedimiento por el cual se construyen edificaciones en tierra. Comúnmente se encontraban edificaciones en tapia pisada en la arquitectura tradicional colombiana. La tierra está compactada dentro de un encofrado en madera (CRAterre, 2015).

4 Adobe: ladrillo de barro y paja, con tamaño variable según las regiones, secado al sol, y cuyo uso es difundido en la edificación de viviendas rurales (Guillaud, Houben, 2006). 


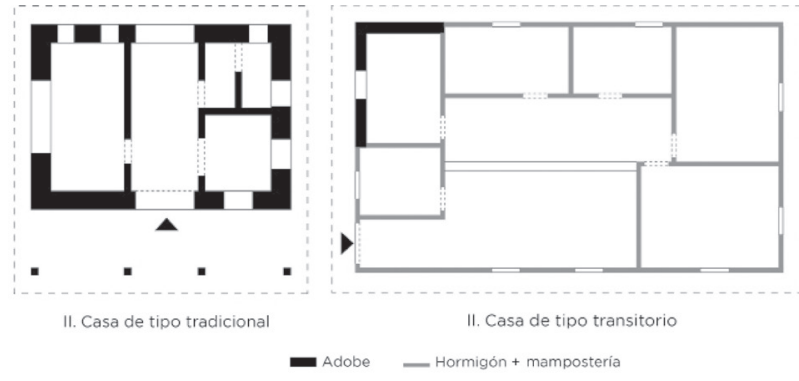

Figura 3. Planos de casas de tipo tradicional y transitorio. Fuente: propia

En Boyacá, las viviendas atípicas comúnmente usan estructuras en hormigón armado, mampostería con ladrillos y techos en fibrocemento. Dichas soluciones no resultan ser adecuadas para conseguir el bienestar al interior de la vivienda dado que la región se caracteriza por un clima tropical seco. El diseño y orientación de las viviendas además de las propiedades térmicas de los materiales, deberían conseguir de minimizar la ganancia y pérdida térmica. Este aspecto ha surgido en variadas entrevistas, donde los usuarios lamentaron temperaturas interiores muy variables a lo largo del día, con excesos (calor o frío) entre día y noche. Sin embargo, en algunas intervenciones recientes donde se ha utilizado el adobe, se demostraron temperaturas constantes obtenidas gracias a la mayor inercia térmica de los muros.

En este caso la investigación se centró en las viviendas típicas, por tanto realizadas con técnicas constructivas tradicionales o autóctonas, aunque actualmente en la región predominan las denominadas construcciones transitorias. Los diversos ejemplos observados tienen en común una planta regular, un único piso y un porche. La organización más común de los espacios interiores consiste en la sucesión de habitaciones, conectadas y accesibles a través del porche que conforma así la fachada principal de la casa. El porche suele ser el espacio principal, siendo flexible, adecuado a diversos usos (reunión, recibo, trabajo, etc.) y permite el control visual de los entornos de la vivienda. Dentro de la vivienda se encuentra la alcoba principal, la cocina, dormitorios y espacios para el depósito de herramientas y otros aprovisionamientos. El baño se ha venido introduciendo recientemente, de hecho se pueden observar dos soluciones espaciales diversas: la primera une un espacio nuevo dentro del volumen principal, la segunda crea un volumen nuevo (no muy lejos del principal), que a efectos de las instalaciones, generalmente se fusiona con el espacio de la cocina.

El diálogo con la comunidad y la estancia in situ, constituyó la base para identificar el camino a seguir en el desarrollo del proyecto. Esto ocurrió tras la identificación de vínculos (de orden cultural, económica y ambiental) que permitieron la determinación de los objetivos, y la definición de un sistema constructivo coherente con éstos y con los recursos locales. De esta manera se direccionó la intervención hacia la realización de casas de un piso, caracterizadas por plantas simples y por soluciones tecnológicas económicas, de fácil ejecución y apropiadas al contexto.

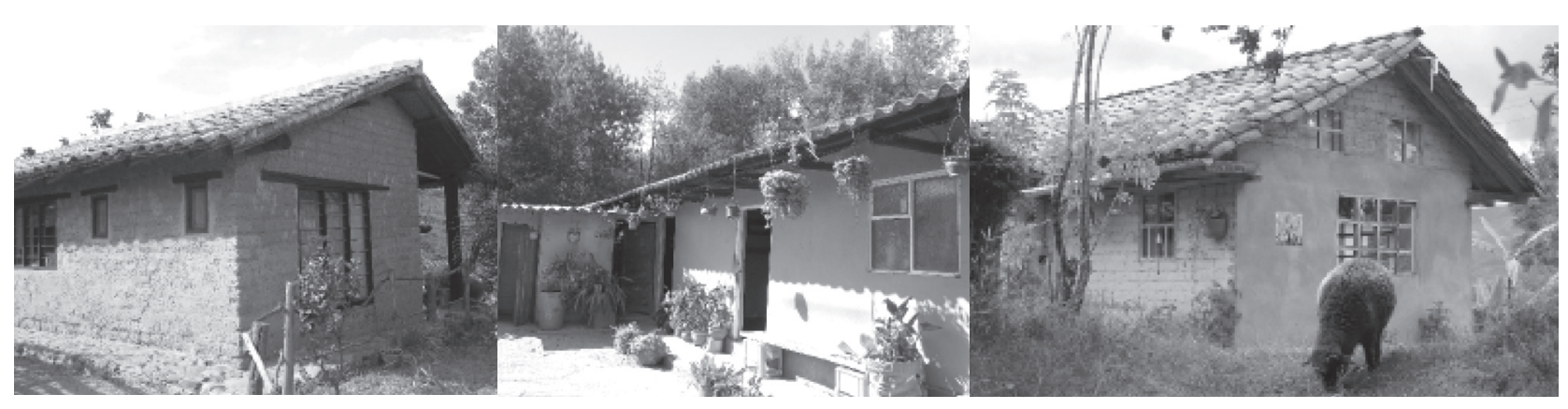

Figura 4. Casas campesinas en Boyacá. Fuente: propia 
Tierra Cruda y Caña brava, desde la tradición hasta la contemporaneidad. Las principales técnicas constructivas tradicionales de la región de Boyacá se fundamentan en el uso de la tierra, caña brava y madera ya que son materiales de alto rendimiento, fácilmente disponibles en gran cantidad, además de ser compatibles con los difundidos procesos de autoconstrucción.

La tierra es un material compuesto, constituido por partes orgánicas e inorgánicas. Su composición influye sobre las características físico-mecánicas y determina le elección de la técnica constructiva más adecuada (adobe, BTC, bahareque, etc.). Su empleo en las construcciones requiere un determinado grado de cohesión que depende de la presencia de conglomerantes naturales (limos y arcillas). Además, cada técnica se caracteriza por específicas mezclas, variables por consistencia, granulometría, plasticidad, presencia de aditivos (paja, cal, arena) y porcentual de agua. (CRAterre, 2015).

La caña brava (Arundo donax) es una planta herbácea que crece en abundancia en toda la región y es característica de la tradición local por sus usos en artesanía y arquitectura. Tiene un crecimiento muy rápido (en un año llega aproximadamente a 5 metros de altura), elevada resistencia mecánica y flexibilidad, y por su vasta difusión en la región resulta ser un material económico.

En las edificaciones rurales se encuentran algunos ejemplos donde la tierra cumple con rol portante (adobe y tapia pisada), en otros casos la función portante se descarga en elementos de madera mientras la tierra tiene rol de relleno (bahareque). Actualmente, las intervenciones de nuevas construcciones con técnicas ancestrales o asociadas a éstas, son más bien limitadas. Dichas técnicas, de hecho han sido suplantadas gradualmente por muros portantes reforzados en ladrillos cocidos o por estructuras de hormigón armado y bloques de ladrillo.

En el departamento se encontraron varias técnicas constructivas, pero en este proyecto se hizo especial referencia a la técnica del bahareque, cuyo significado literal es "pared de caña y tierra". En el bahareque la estructura portante es en madera (con luces de 120/180 centímetros) al interior de las cuales se teje una estructura secundaria con elementos horizontales (en caña brava o madera) de soporte para las capas de barro que completan y acaban la pared (Van Lengen, 2003) (Ver figura. 5). Esta técnica, típica de la tradición rural boyacense, fue progresivamente sustituida por construcciones en cemento y ladrillos, a pesar de que el costo sea excesivo para la mayoría de la población campesina. Investigando sobre la caída en desuso del bahareque se descubrió que estuvo relacionada con problemas de insalubridad e inestabilidad estructural, por tanto resultando menos adecuada respecto a otras técnicas. Comúnmente se han asociado estos problemas con un defecto del material o de la técnica constructiva y se ha considerado el bahareque una solución pobre, sinónimo de indigencia, para la edificación de viviendas rurales. Sin embargo la mayoría de los problemas encontrados en el tiempo fueron debidos a errores ejecutivos que, hoy en día se pueden evitar integrando los saberes ancestrales con los avances en materia de construcción y tecnología, junto con los resultados de las varias investigaciones científicas en el tema de arquitectura en madera y barro.

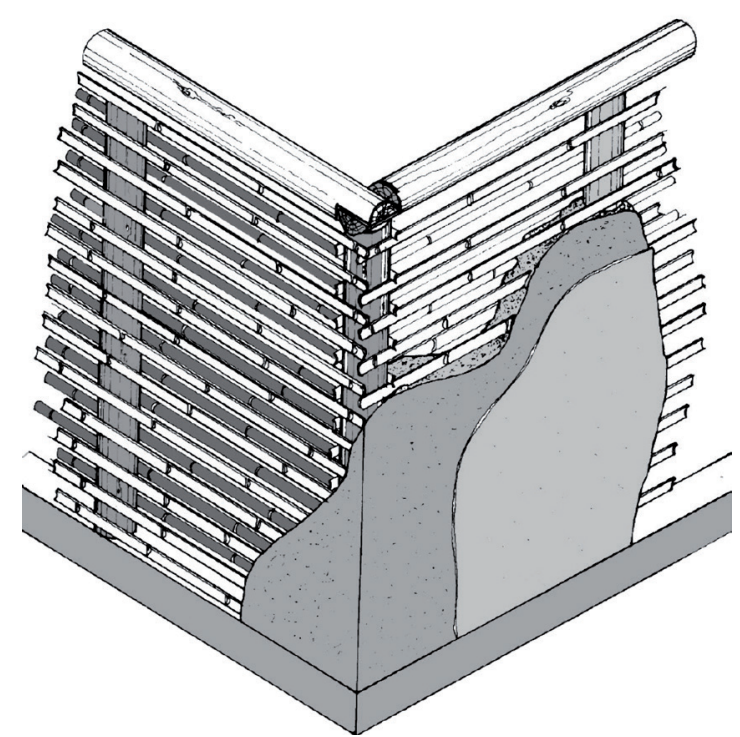

Figura 5. Proceso ejecutivo del bahareque. Fuente: Carazas Aedo \& Rivero Olmos (2002) 


\section{Resultados: el panel MTC}

Con estos presupuestos, el proyecto inició con la realización de un prototipo de panel desde el cual se desarrolló el panel M.T.C. De hecho, con el prototipo se dio un enfoque práctico a la investigación con el propósito de conocer los diversos materiales empleados, sus propiedades físico-mecánicas y evaluar la posibilidad de combinación entre ellos para crear un panel modular (Figura 6). El estudio de la caña brava se profundizó con algunas visitas a los cultivos para conocer el proceso productivo (desde el cultivo hasta el corte y manipulación) y a través de cursos de capacitación con los artesanos locales se comprendió el proceso de producción y se observó su aplicación en ejemplos de arquitectura tradicional y contemporánea. En las nuevas intervenciones su aplicación interesa principalmente en los techos, donde las cañas están atadas entre ellas y soportadas por viguetas de tal manera que conformen un plano de soporte para una capa de barro y tejas.

El estudio de la tierra se llevó a cabo a través de la realización de diversas mezclas que varían según el uso en la construcción. De hecho el barro tiene porcentajes variables de tierra y agua, al cual se pueden añadir varios elementos (cal, arena, fibras vegetales etc.) con el objetivo de mejorar sus características. La mezcla utilizada en el bahareque se compone de tierra, cal, arena en las siguientes proporciones 7-1-1 añadiendo fibras vegetales (fique o paja) y agua hasta obtener una pasta blanda y bastante líquida. Durante la fase de secado la mezcla está sometida a tensiones debidas a la evaporación del agua, por tanto pueden formarse grietas (aún más cuando el secado ocurre rápidamente). Para contrastar estas tensiones y conseguir mayor estabilidad al material se añaden las fibras vegetales.

El contexto de intervención, con sus características climáticas y económicas, ha condicionado la elección de los materiales y el diseño del panel permite aprovechar de las ventajas y contrastar las debilidades de cada uno de los materiales. Así, tomando por ejemplo la técnica del bahareque caracterizada por tramas regulares, se desarrolló la hipótesis de un sistema modular conformado por paneles con los mismos materiales. Los paneles, independientes cuando llegan en obra, se ensamblan en seco con tornillos.

La estructura principal es un marco de madera que mide 120x240x15 cm y tiene rol estructural por ser un material de alta resistencia, fácilmente disponible en la región y cuya manipulación es difundida entre los artesanos locales. La madera elegida fue el pino que crece en varias partes del departamento de Boyacá. La unión entre los elementos verticales y horizontales del marco se resolvió con tornillos por ser de simple ejecución además de conseguir uniones rígidas (Figura 7).

Dentro del marco se colocó una estructura secundaria en caña brava, aprovechando que este material crece en toda la región, es económico e igualmente su manipulación es simple y difundida entre los artesanos. Primero se colocaron cinco cañas verticalmente, de modo que actúen como soporte para el sucesivo entramado. Las cañas fueron conectadas a la estructura de madera a través de tornillos que entran en sus cavidades. El entramado se realizó con cañas cortadas en cuartos (Figura 8), apoyadas en alternancia a las cañas verticales (cuya sección es entera). Este entramado permitió rigidizar el panel frente a los esfuerzos

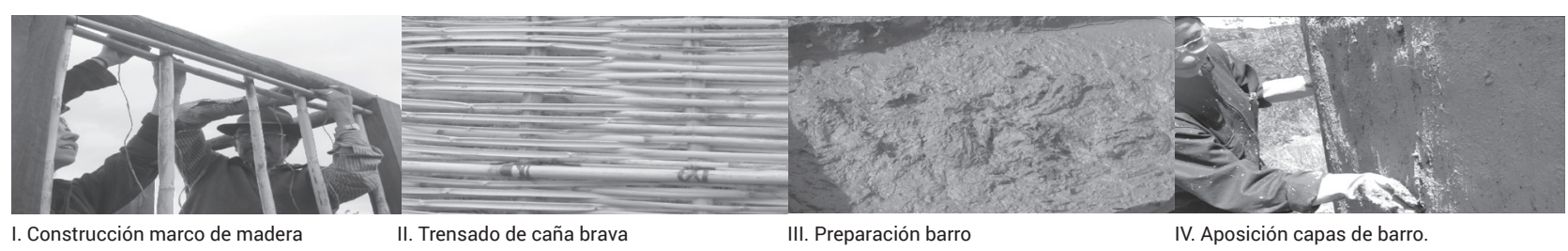

Figura 6. Construcción del prototipo: Fuente: propia 

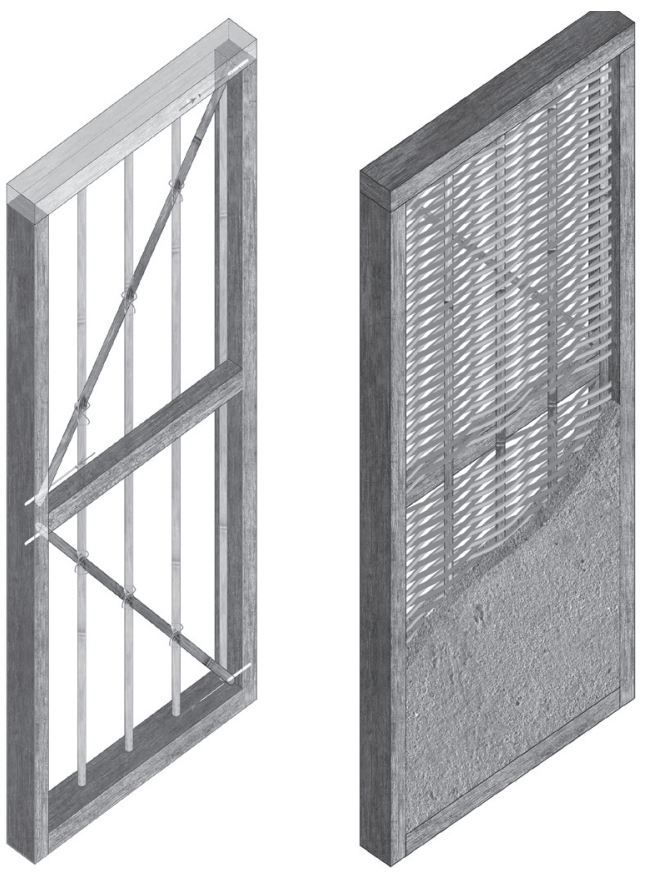

Figura 7. Panel M.T.C. Esquemas de la estructura en madera, caña y barro. Fuente: propia

y creó un necesario soporte para la aposición de barro. Las cañas del entramado quedan fijas por tensión (haciendo el entramado, los elementos se curvan como arcos y la tensión impide que se deslicen desde los soportes verticales). Además se introdujeron riostras en cañas (con sección entera) que trabajan a tracción para contrastar las acciones horizontales en caso de sismo. Estos elementos diagonales se ataron al entramado con varios amarres en fibra de fique de manera que no se verifique el pandeo.

El fique y la caña brava son vulnerables frente a varios agentes atmosféricos, por ello el recubrimiento con las capas de barro permite protegerlos del deterioro además de garantizar la suficiente inercia térmica al panel (Minke, 2010).

El tamaño modular responde a la necesidad de simplificar la construcción de casas a través de procesos de autoconstrucción, limitando los gastos con la utilización de barras de madera que se encuentran en el comercio. Con estas dimensiones el marco tiene capacidad portante y

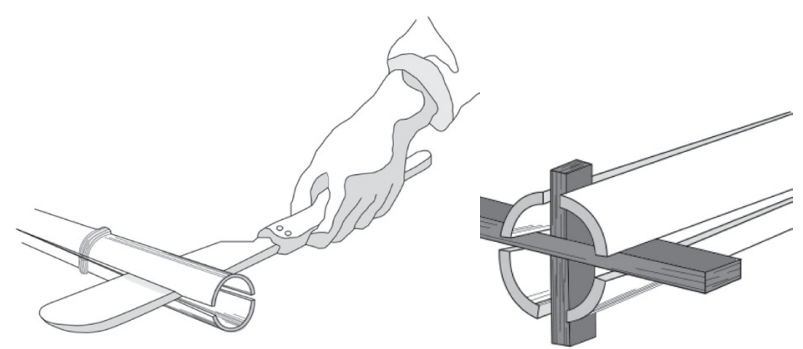

Figura 8. Corte de las cañas para realizar el entramado. Fuente: Van Lengen (2003)

limita su uso a lo necesario estructuralmente. La modularidad permite además utilizar el mismo panel en cubierta y paredes interiores. Desde un panel básico (enteramente entramado) se propusieron otras dos variantes; un panel para colocar la ventana y otro para la puerta. El panel hasta su fase prefabricada (en madera y caña) tiene un peso de $50 \mathrm{~kg}$ y es por tanto fácilmente transportable a la obra además de ser movible por dos personas.

La mezcla de barro puede realizarse con la tierra extraída directamente en la obra, a la cual se añade cal, arena, fique y agua según las mismas proporciones del bahareque. La masa se aplica manualmente en tres capas, dejando algunos días de secado progresivo entre cada capa. Es fundamental que el secado ocurra de manera progresiva de tal manera que se evite la formación de grietas. Para mejorar la resistencia frente al agua, la mezcla puede ser integrada por aditivos (gel de nopal ${ }^{5}$ ) o se puede acabar la pared con una capa de yeso con lechada de cal (Salas Serrano, 1995).

En síntesis, el sistema constructivo prevé dos fases de realización, la primera por parte de artesanos (prefabricación) y la segunda por parte de los usuarios (autoconstrucción). La prefabricación llega hasta la realización de un panel modular y portante en madera y caña brava. Después de unir los paneles con la cimentación y entre ellos se completa la obra en autoconstrucción tras la

5 Nopal: También denominada chumbera o tuna, es una planta de la familia de las cactáceas (Cactaceae) y su nombre científico es Opuntia ficus-indica (Mondragón-Jacobo y Pérez González, 2003). 
aposición de tres capas de barro. Para prevenir el deterioro del barro por agentes atmosféricos externos, se apoyan los paneles sobre una base de piedra y hormigón (cimentación ciclópea) separándolos del terreno. La unión entre los paneles se hace mediante atornillados con el fin de obtener una alta robustez del sistema y la resistencia necesaria a diversos esfuerzos mecánicos.

Para comprobar las características mecánicas y térmicas del panel se hicieron algunos análisis que simularon las condiciones de uso en una vivienda rural situada en Ráquira. El análisis estructural se realizó con el software "Straus" en el que se dibujó un modelo del panel M.T.C. cargado horizontalmente y verticalmente según los datos calculados en el proyecto de la vivienda. Los resultados de este modelo se compararon con los resultados de otro modelo sin entramado en caña brava (solo el marco en madera). En presencia de cargas verticales los esfuerzos resultaron soportados principalmente

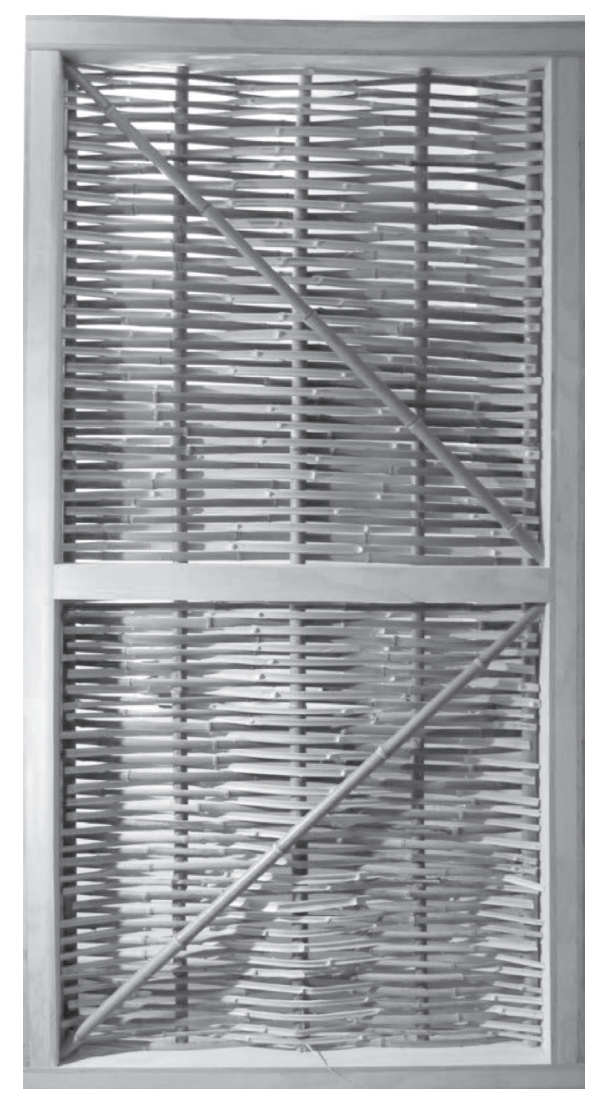

Figura 9. Modelo escala 1:1 del Panel M.T.C. Fuente: propia por la estructura en madera. El entramado en caña brava permitió reducir la flexión del larguero superior en madera. Con cargas horizontales se intentó simular la acción sísmica y se demostró el importante aporte de las riostras en caña brava. De hecho, el modelo sin entramado en caña brava, tiene desplazamientos veinte veces mayores respecto al panel M.T.C.

Para el análisis térmico se recolectaron todas las informaciones climáticas de la región y los datos físicos de los materiales. Con el programa "Bistra" se pudo realizar una simulación dinámica del panel (a lo largo de un día entero). Los valores de transmitancia térmica periódica y desfasamiento permiten decir que el uso del panel podría significadamente mejorar las condiciones climáticas interiores de las viviendas, sobre todo cuando se ponen en comparación con los valores obtenidos para construcciones en mampostería de ladrillo.

\section{Conclusiones}

El Departamento de Boyacá tiene abundantes recursos naturales y conocimientos ancestrales sobre sus usos. A nivel nacional se observa una gran innovación en los materiales y las técnicas constructivas con la introducción de métodos industrializados para responder a un fenómeno de rápido crecimiento urbano. El uso de estos materiales está fuera del alcance de la mayoría de los campesinos (evidenciado por los datos DANE sobre la pobreza en zonas rurales) y resulta en la edificación de casas que no responden a las necesidades además de presentar carencias a nivel de seguridad y salubridad.

La investigación llevada a cabo ha demostrado cómo el uso de los recursos locales y de los conocimientos ancestrales permite desarrollar soluciones innovadoras, coherentes con el contexto y adecuadas a las exigencias de la población rural. De ahí que el presente trabajo hable sobre la utilización de materiales locales y la reintroducción de métodos constructivos tradicionales, para lograr una síntesis entre tradición e innovación. El 


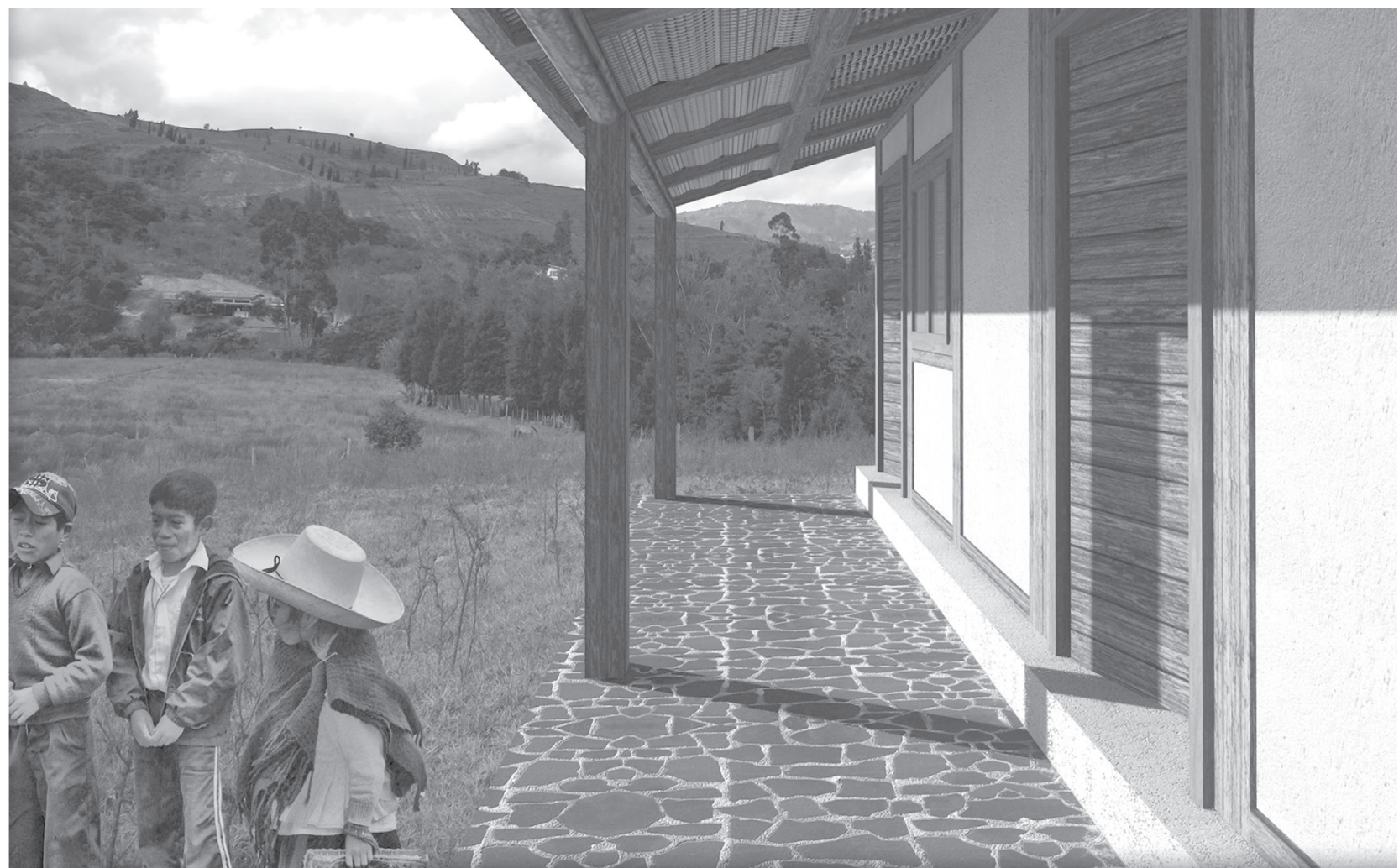

Figura 10. Ilustración del proyecto de una vivienda con Panel M.T.C. Fuente: propia

sistema de paneles M.T.C. se basa en la ejecución, por mano de obra local de un proceso tradicional (bahareque), aplicando al mismo tiempo nuevas tecnologías para conseguir una vivienda sostenible. Se consigue mejorar la calidad ambiental y al mismo tiempo se valoriza la labor local y el uso consciente de los recursos naturales. Hasta el momento la investigación ha logrado responder a los objetivos inicialmente evidenciados, $y$ ha demostrado la posibilidad de alternativas constructivas en la realización de nuevas viviendas en zonas rurales.

El sistema del Panel M.T.C. abre el camino para otros desarrollos, siendo ahora un proyecto experimental (Figura 10.) limitado a las zonas rurales de Boyacá. Dada la gran existencia de materiales (tierra y madera) se podría aplicar esta solución en otras zonas geográficas, y en ausencia de caña brava se podrían utilizar las bambuseae, presente en casi todas las zonas tropicales del mundo. Las diferencias de clima pueden requerir que el panel esté compuesto por más capas (incluyendo una capa aislante o ventilada), así como en regiones más lluviosas se necesitaría buscar soluciones de impermeabilización del barro. También en contextos donde los transportes sean más eficientes y menos dificultosos se podría prefabricar el panel por entero, con importantes beneficios en tiempos de ejecución.

\section{Referencias bibliográficas}

Cuadrado Mulero, A. (2013). Construcción de cerramiento con Paneles de Quincha - Acabados de adobe y cal, España.

DANE, Proyecciones nacionales y departamentales de población 2005 -2020, Boyacá, Universidad Santo Tomas.

Fonseca Martínez L., Saldarriaga Roa A. (1980). La arquitectura de la vivienda en Colombia, Bogotá, Colciencias. 
Fonseca Martínez L. \& Saldarriaga Roa, A. (1992). Arquitectura popular en Colombia: Herencias y tradiciones, Bogotá, Altamir Ediciones.

Guillaud H., Houben H., Dethier J., CRATerre, (2006). Traité de construction en terre, Grenoble, Parenthèses.

Gálligo P., (2005). Un techo para vivir - Tecnologías para viviendas de producción social en América Latina, Barcelona: Universidad Politécnica de Catalunya.

Minke G. (2010). Manual de construcción en tierra - La tierra como material de construcción y su aplicación en la arquitectura actual, Spagna, EcoHabitar Olba-Teruel.
Mondragón-Jacobo C., Pérez González S., (2003). El nopal como forraje, Universidad Autónoma de Querétaro, México, Instituto Nacional de Investigaciones Forestales y Agropecuarias.

Navarrete S., (2015). Guía metodológica: Cultivo y uso de la caña brava para cerramientos arquitectónicos, Bogotá, Universidad El Bosque.

Salas Serrano J. (1995) Habiterra: Exposición iberoamericana de construcciones de tierra, Santafé de Bogotá, Escala.

Saldarriaga Roa A. (2013). Hábitat rural y vivienda campesina en Colombia, Bogotá, Fundación Corona.

Van Lengen J. (2003) Manual del arquitecto descalzo, Bogotá, Alfaomega colombiana. 